\title{
Desarrollo de estrategias para integración de las Redes Verticales de América del Sur con base en los términos de referencia SIRGAS/GGOS/IAG
}

\author{
Andrea Galudht Santacruz Jaramillo*
}

Recibido el 4 de junio de 2017; aceptado el 11 de octubre de 2017

\section{Resumen}

Los Sistemas Verticales de Referencia (SVRs) junto con sus materializaciones nacionales, regionales y globales, se encuentran en una evolución significativa en los últimos años debido a que son fundamentales para el monitoreo de procesos físicos asociados a cambios en el Sistema Tierra. El aval de los sistemas de observación Terrestre proviene del Global Geodetic Observing System (GGOS) soportado por la International Association of Geodesy (IAG). Estas organizaciones se basan en estándares y términos de referencia para generar información geoespacial precisa y confiable. El Sistema de Referencia Geocéntrico para las Américas (SIRGAS), referente a su Grupo de Trabajo III: Datum Vertical, busca atender las exigencias de GGOS/IAG para proporcionar un Sistema Vertical de Referencia SIRGAS (SVRS) moderno. Para esto se debe homogeneizar y unificar las Redes Verticales de Referencia Nacionales (RVRNs) considerando el campo de la gravedad. Esta investigación presenta la metodología desarrollada para un inventario de metadatos de la RVRN del Ecuador en vista de los términos de referencia de SIRGAS/GGOS/IAG, el cual puede servir de base para el desarrollo de estrategias para integrar las Redes Verticales de América del Sur. Además, este inventario contribuye a la futura creación de una Infraestructura de Datos Espaciales para las Américas, según los objetivos de UN-GGIM: Américas.

Palabras clave: Sistemas y Redes Verticales de Referencia Nacionales y Global, Inventario, IDE, SIRGAS, GGOS, metadatos.

* Universidade Federal do Paraná (UFPR), Setor de Ciências da Terra, Departamento de Geomática, Programa de Pós-Graduação em Ciências Geodésicas, Rua Cel. Francisco Heráclito dos Santos 210, Jardim das Américas, Curitiba, PR, Brasil, 81531-970, e-mail: andrea.santacruz@ufpr.br 


\section{Resumo}

Os Sistemas Verticais de Referência (SVRs) junto com suas materializações nacionais, regionais e global, se encontram em uma evolução significativa nos últimos anos, a qual é fundamental para o monitoramento de processos físicos associados a mudanças no Sistema Terra. O aval dos sistemas de observação Terrestre vem do Global Geodetic Observing System (GGOS) suportado pela International Association of Geodesy (IAG). Estas organizações baseiam-se nos padrões e termos de referência para gerar informação geoespacial precisa e confiável. O Sistema de Referência Geocêntrico para as Américas (SIRGAS), específicamente no Grupo de Trabalho III: Datum Vertical, busca atender as exigências de GGOS/IAG para proporcionar um Sistema Vertical de Referência SIRGAS (SVRS) moderno. Para isso deve-se homogeneizar e unificar as Redes Verticais de Referência Nacionais (RVRNs) considerando o campo da gravidade. Esta investigação apresenta a metodologia desenvolvida para um inventário de metadados da RVRN do Equador em vista dos termos de referência de SIRGAS/GGOS/IAG, que poderá servir de base para o desenvolvimento de estratégias para integração das Redes Verticais da América do Sul. Além disso, este inventário contribui para uma futura criação de uma Infraestrutura de Dados Espaciais para as Américas, segundo os objetivos do UNGGIM: Américas.

Palavras chave: Sistemas e Redes Verticais de Referência Nacionais e Global, Inventário, IDE, SIRGAS, GGOS, metadados.

\section{Abstract}

The Vertical Reference Systems (VRSs) along with their national, regional and global materializations in frames, have been in a significant evolution in the last years because they are fundamental for the monitoring of physical processes associated with changes in the Earth System. The endorsement of Earth observation systems comes from the Global Geodetic Observing System (GGOS) supported by the International Association of Geodesy (IAG). These organizations are based on standards and reference terms to generate accurate and reliable geospatial information. The SIRGAS (Sistema de Referencia Geocéntrico para las Américas), in the context of its Working Group III: Vertical Datum, aims to meet the requirements of GGOS/IAG to provide a modern SIRGAS Vertical Reference System (SVRS). For this, it is necessary to homogenize and unify the National Vertical Reference Networks (NVRNs) considering the gravity field. This research presents the methodology developed for an Ecuadorian NVRN metadata inventory in view of the SIRGAS/GGOS/IAG reference terms. The achieved results can serve as a basis for the development strategies for the integration the Vertical Networks in South America. Also, this inventory contributes for building a future Spatial Data Infrastructure for the Americas according the objectives of the UN-GGIM: Americas. 
Keywords: National and Global Vertical Reference Systems and Frames, Inventory, SDI, SIRGAS, GGOS, metadata.

\section{Contribución del trabajo}

Uno de los objetivos de este artículo es resumir el trabajo realizado en la tesis de maestría de Santacruz (2015), con la cual se generó el artículo de Santacruz Jaramillo et al. (2015). Es importante recalcar que este tema ha sido bastante debatido durante estos últimos años y que hasta la época ya se han generado nuevos estudios, convenciones y resoluciones acerca de Redes Verticales, tal como es presentado, por ejemplo, en De Freitas (2015), Ihde et al. (2015), Drewes et al. (2016) y Sánchez y Sideris (2017). Se destaca la relevancia del tema propuesto, el cual es recomendado en Angermann et al. (2016), en el ítem referente a Sistemas de Alturas y sus realizaciones. Considerando los antecedentes presentados, el presente trabajo realiza la vinculación de dos temas relevantes en el ámbito de la Geodesia y de la Información Geoespacial. Por lo tanto, se puede señalar que existen dos importantes contribuciones. La primera con ámbito en la Geodesia, la cual es la propuesta de una metodología para la generación de estrategias de solución para la integración de las redes verticales de América del Sur. Esta metodología puede ser base para la búsqueda del análisis de los datos y metadatos de cada país, y puede ser modificada dependiendo de sus propias condiciones. La segunda contribución se refiere al uso de datos geoespaciales estandarizados, lo cual desencadena una serie de necesidades futuras que atienden a la creación o actualización de Base de Datos y Metadatos vinculados a una IDE. Esto, permitirá el intercambio y la interoperabilidad de datos con la finalidad de facilitar información al usuario, específicamente enfocado al almacenamiento de datos geodésicos de SIRGAS.

\section{Estado del Arte}

A nivel mundial, existen organizaciones y servicios científicos que buscan generar geoinformación con precisión y confiabilidad necesaria para aplicaciones en los más diversos campos de actividades. Estas informaciones de forma específica y en función de su aplicabilidad, son las necesarias para el desarrollo de estudios y proyectos focalizados en la solución de problemas referentes a la dinámica del Sistema Tierra, englobando investigaciones en las diferentes ramas de la Geodesia. Actualmente, la visión de la Geodesia está direccionada hacia el uso de diferentes y modernas herramientas para la obtención de información espacialmente referenciada.

La Asociación Internacional de Geodesia (IAG) es la organización que se dedica a la coordinación de todas las actividades de la Geodesia en el planeta y, en especial, tiene un enfoque central en la investigación de frontera de esta Ciencia. La IAG 
promueve la cooperación científica, avance e investigación en Geodesia en escala global y genera contribución a través de varios organismos de investigación (IAG, 2014). Los objetivos se enfocan en el estudio de todos los problemas geodésicos relacionados con la observación de la Tierra asociados a los cambios globales. La IAG se encuentra estructurada en diferentes Servicios científicos, Comisiones, Subcomisiones e Inter-comisiones, que permiten generar investigación en ramas específicas como: Sistemas de Referencia; Campo de la Gravedad Terrestre; Rotación de la Tierra y Geodinámica; Posicionamiento y sus aplicaciones. Fue creada la InterComisión 1.2 (IAG Inter-Commission Project 1.2: Vertical Reference Frames -IAG ICP 1.2) con el propósito de establecer convenciones para la definición del Sistema Global de Alturas - SGA (conocido en inglés como World Height System —WHS) o Sistema Vertical de Referencia Global —SVRG (Global Vertical Reference System -GVRS) y su materialización a través de la Red Vertical de Referencia Global -RVRG (Global Vertical Reference Frame - GVRF) basado en el geopotencial. Dicha información puede ser consultada en Ihde et al. (2007): Conventions for the Definition and Realization of a Conventional Vertical Reference System (CVRS). Para cumplir con este objetivo, fue necesaria la creación de varios proyectos de apoyo, para dar continuidad con los objetivos y para evaluar estas convenciones. En 2011 la IAG, integró los trabajos designados a la IAG ICP 1.2 al proyecto Global Geodetic Observing System (GGOS) con el objetivo de recopilar los datos geodésicos globales para generar una infraestructura y base científica interoperable que servirá en los estudios referentes al cambio global en Ciencias de la Tierra. GGOS tiene como visión el avance y la continuidad de los estudios referentes a la dinámica del sistema Tierra relacionando la cuantificación de los cambios globales en espacio y tiempo. Por lo tanto, providencia una infraestructura geodésica precisa para el monitoreo del Sistema Tierra (PLAG Y PEARLMAN, 2009). Con un enfoque en la importancia para la ciencia y la sociedad del desarrollo de temas que integren grupos interdisciplinarios, GGOS dividió sus investigaciones en tres temas. Siendo el Tema1: Estandarización de un Único Sistema Global de Alturas o SVRG, el foco de esta investigación. El objetivo central es proporcionar un Sistema Vertical de Referencia Global relacionado al campo de la gravedad global, el cual:

- Sea compatible con el ITRS;

- Tenga una componente física con precisión de (1 ppb) 1 cm en las coordenadas verticales (Plag y Pearlman, 2009);

- Permita la unificación de todos los Sistemas Verticales de Referencia Nacionales (SVRNs) existentes;

- Garantiza coordenadas verticales con una consistencia global y estabilidad a largo plazo (Kutterer et al., 2012). 
Siguiendo la línea de investigación, el Sistema de Referencia Geocéntrico para las Américas (SIRGAS) forma parte de la Comisión 1 de la IAG y pertenece al Grupo de Trabajo de la Comisión de Cartografía del Instituto Panamericano de Geografía e Historia (IPGH), el cual corresponde a la Organización de los Estados Americanos (OEA). Esta organización es la base para el desenvolvimiento de proyectos que tienen como objetivo la generación y uso de información georreferenciada en América del Sur, América Central y El Caribe. Dentro de SIRGAS se establecieron grupos de trabajo. El Grupo de Trabajo III: Datum Vertical (SIRGAS-GTIII), tiene nuevos objetivos relacionando términos de referencia de GGOS, que actualmente se encuentran en la vanguardia del conocimiento, los cuales permitirán tener la base para la integración de los SVRNs y con esto, el establecimiento de un Sistema Vertical de Referencia SIRGAS (SVRS), relacionado con el campo de la gravedad que sea completamente consistente, con estabilidad en el tiempo, homogeneidad y alta fiabilidad. La componente geométrica tiene como superficie de referencia el datum SIRGAS (elipsoide geocéntrico del Geodetic Reference System 1980 - GRS80) y alturas elipsoidales como coordenadas. La componente física del nuevo sistema de referencia vertical incluye: nivelaciones de primer orden, valores de gravedad, números geopotenciales, registros mareográficos y datos de altimetría satelital. Además, se define por un nivel global unificado de referencia $W_{0}$ y números geopotenciales como coordenadas verticales fundamentales (Sánchez, 2009; SIRGAS, 2014). El nuevo Sistema Vertical de Referencia SIRGAS se establecerá sobre los términos de referencia de GGOS/IAG, los cuales darán al sistema homogeneidad, estabilidad a largo plazo y alta confiabilidad.

A través de la Figura 1, se observa en forma general los lineamientos para establecer el SVRS, el cual depende de las metodologías de integración de todos los SVRNs y de las convenciones del SVRG.

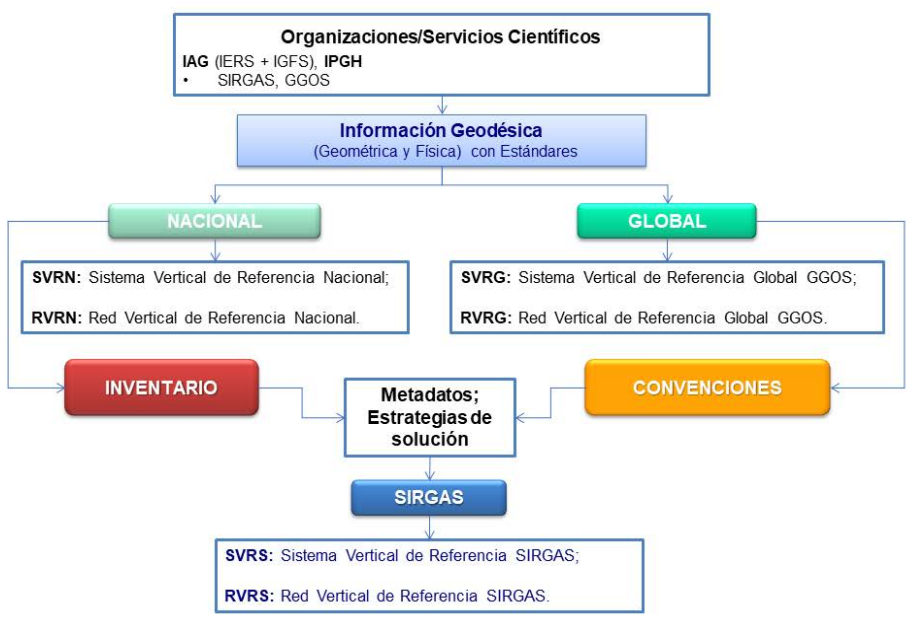

Figura 1. Establecimiento del SVRS.

Fuente: Santacruz, 2015. 
Para atender este objetivo, diversos autores como De Freitas et al. (2002), Drewes et al. (2002), Sánchez y Luz (2011) entre otros, comenzaron con el estudio de cómo hacer posible esta integración. Considerando las contribuciones de los autores, se visa la necesidad de implementar o adaptar a las condiciones locales, convenciones globales como complemento. Dichas convenciones globales fueron creadas para la definición y realización de un SVRG (Ihde et al., 2007). Por esta razón, se usa los términos de referencia de la SIRGAS/GGOS/IAG (Kutterer et al., 2012), que dan directrices sobre la unificación de un SVRG y que actualmente es la base para todo tipo de investigación que envuelve el estudio de la dinámica terrestre y su aplicabilidad a la sociedad.

Considerando estos puntos, se realza la importancia que tiene las relaciones entre organizaciones con fines comunes. En este caso, el de buscar la unificación de las RVRNs para el establecimiento de un SVRS con una vinculación futura a un Sistema Vertical de Referencia Global y la estandarización y difusión de los datos y metadatos interoperables, para generar herramientas de acceso para el usuario. El problema de esta unificación se basa en la incompatibilidad de los datos existentes de las redes nacionales debido a que su establecimiento fue realizado con diversas metodologías, bajo diferentes data verticales, con épocas distintas de realización. En general, estas realizaciones no consideran aspectos geodinámicos, entre otros factores importantes para ser considerados. Con lo expuesto, se puede considerar que las redes verticales presentan considerables discrepancias entre países, las cuales no permiten el intercambio consistente de geoinfomación. Por lo tanto, no son aptos para la determinación de alturas a partir de técnicas GNSS (Global Navigation Satellite System) con combinación de los datos generados a través de los MGGs (Modelos Globales del Geopotencial o Global Geopotential Models -GGMs), limitando así, la vinculación al SVRG. Otro problema de incompatibilidad se genera debido a la falta de organización y homogenización de los datos de las RVRNs, específicamente, la ausencia del almacenamiento de bases de datos estandarizadas.

Para poder desarrollar estrategias para la generación de posibles alternativas de conexión de las redes verticales pertenecientes a los países miembros de SIRGAS, es necesario estudiar cada una de las redes nacionales y recopilar toda la información existente de estas. La base de esta investigación es generar un inventario estandarizado basado en metadatos, que reúna tanto los estudios anteriormente realizados como los nuevos, con la visión de reunir todas las características específicas de las Redes Verticales y de las Redes Gravimétricas para la generación de estrategias nacionales. La modernización e integración de los SVRNs junto con el empleo de las convenciones para el establecimiento del SVRG, darán la pauta para la definición de un único SVRS con su respectiva Red Vertical de Referencia SIRGAS (RVRS), basada en diferencias de números geopotenciales consistentes 
para el continente. Actualmente, este es el paso primordial para SIRGAS-GTIII, desde el punto de vista moderno de la Geodesia, para poder realizar la integración de cada una de las redes nacionales hacia una única Red Continental enlazada a un SVRG.

Con este trabajo se busca el establecimiento de estrategias, mediante la contribución y protagonismo de los países miembros de SIRGAS en el desarrollo, implementación y continuidad de este inventario. Esto trae consigo ventajas respecto a una visión futura del acceso y descentralización de la información, ya que provocará la necesidad de creación de una única Infraestructura de Datos Espaciales (IDE) para SIRGAS. Esta IDE atenderá los objetivos de UN-GGIM: Américas (UNGGIM AMÉRICAS, 2014), la cual permitirá la interoperabilidad e intercambio de geoinformación entre las posibles IDEs nacionales y globales, lo cual facilitará el uso de este tipo de información geodésica, atenderá los principios de uso de información abierta y el uso de software libre para beneficio del usuario y para el desarrollo de nuevas aplicaciones para bien de la sociedad.

\section{Problema del Valor de Contorno de la Geodesia (PVCG) y números geopotenciales}

La ecuación de Laplace $\Delta \nabla_{e x t}=0$ considera la distribución de las masas en un punto exterior a las mismas, por lo tanto, no considera densidades (Gemael, 1999). El PVCG relaciona la condición de Laplace para la determinación del campo de la gravedad externo terrestre, a través del cálculo de una función armónica en el exterior de una superficie de contorno $S$ desconocida (Hofmann-Wellenhof y Moritz, 2005).

Con la nueva visión de la Geodesia, el estudio del segundo PVCG es relevante en la determinación del Geopotencial W, considerando conocidos la geometría de la superficie de la Tierra y la gravedad sobre esta, variando la condición de contorno.

En las redes de nivelación clásicas, la determinación de $W_{P}$ en un punto $\mathrm{P}$ es dada por:

$$
W_{P}=W_{0_{\mathrm{i}}}+C_{P}
$$

En las Redes clásicas $W_{0 i}$ es un valor arbitrado en los Data Verticales de cada país. Y el número geopotencial $C_{P}$ en el punto $P$ es:

$$
C_{P}=W_{0_{i}}-W_{P}=\int g d n \cong \sum g_{m_{i}} \Delta_{m_{i}}
$$

La integral de la ecuación (2) representa la forma clásica práctica para la determinación de números geopotenciales con desniveles y el valor medio de la gravedad entre los extremos de una sección de nivelación. Y la primera parte de esta 
misma ecuación figura la forma moderna para la determinación de números geopotenciales sin la necesidad de depender de nivelación, y si de datos gravimétricos y dados GNSS para su cálculo. Para aplicar esta forma, es necesario el uso del PVCG y la determinación de varios funcionales para el cálculo de $W_{P}$.

Para el cálculo de $W_{P}$ es necesario la determinación del potencial perturbador $T_{P}$ así como el uso del potencial normal $U_{P}$ :

$$
W_{P}=U_{P}+T_{P}
$$

$U_{P}$ es dado por:

$$
U_{P}=U_{0}+\frac{\partial U_{0}}{\partial h} h_{P}
$$

Obtenido de $U_{0}$, que es el potencial normal de la gravedad en el elipsoide de referencia. Por otra parte, $T_{P}$ pode ser calculado segundo Heck (2011), por una solución de primer orden para el PVCG. Esta determinación es basada en la teoría de Molodensky, utilizado la solución por Brovar (1972):

$$
T_{P}=\frac{R}{4 \pi} \iint_{S}\left(g_{P}+g_{1}=+\cdots\right) H(\psi) \cdot d s
$$

Donde $\delta_{g}$ es el disturbio de la gravedad, $R$ es el radio medio de la Tierra y $H(\psi)$ es la función de Hotine-Koch, la cual es presentada en Hofmann-Wellenhof y Moritz (2005), pp.115.

Para la determinación de $T_{P}$ es necesario el cálculo del $\delta_{g}$ dado por la gravedad normal y la gravedad observada en el punto:

$$
\delta_{g_{P}}=g_{P}-\gamma_{P}
$$

Para que el resultado de $T_{P}$ sea más refinado, puede ser determinado el $\delta g$ residual a través de descomposición espectral y el uso de Modelos Globales del geopotencial, así como también de Modelos Digitales de Altura.

\section{Estándares de la Información}

La Organización Internacional para la Normalización (International Organization for Standardization - ISO) es responsable de planear, formular y adoptar Normas Internacionales (ISO/TC 211, 2014). 


\section{EI ISO/TC 211 Información Geográfica / Geomática}

Tiene como alcance la normalización en el campo de la información geográfica digital. Pretende establecer un conjunto estructurado de normas de información relativa a los objetos o fenómenos directa o indirectamente relacionados con una localización en relación con la Tierra. El ISO/TC 211 cuenta con una comunidad de usuarios, entre ellos se encuentra la iag y el ipgh, lo cual expresa la importancia que tiene la normalización de datos espaciales (ISO, 2003. Traducción: IPGH, 2010; ISO/TC 211, 2014).

Para desarrollar la investigación propuesta, se consideró la Norma ISO 19115: 2003 Información Geográfica - Metadatos y la ISO 19139 para la determinación de Conjunto de Datos Geográficos (CDG), perfil de metadatos, plantilla de metadatos. También fueron creados diagramas de clase de uso y de clases para un mejor entendimiento y desarrollo del trabajo.

Respecto a los Metadatos, por definición según UN-GGIM: Américas (2013), son el conjunto de información que describe los datos, incluyendo las características de su levantamiento, producción, calidad y estructura de almacenamiento, y lo que es esencial para promover su documentación, integración y despliegue, así como permitir su búsqueda y exploración. Todas las características obtenidas de los datos son base tanto para la identificación de posibles estrategias de solución, así como para un futuro establecimiento de una IDE. Basados en las descripciones anteriores, tenemos como resultado los metadatos recopilados que describen la parte de la Geodesia, así como la parte de Información Geoespacial, tal como se muestra en la Figura 2.

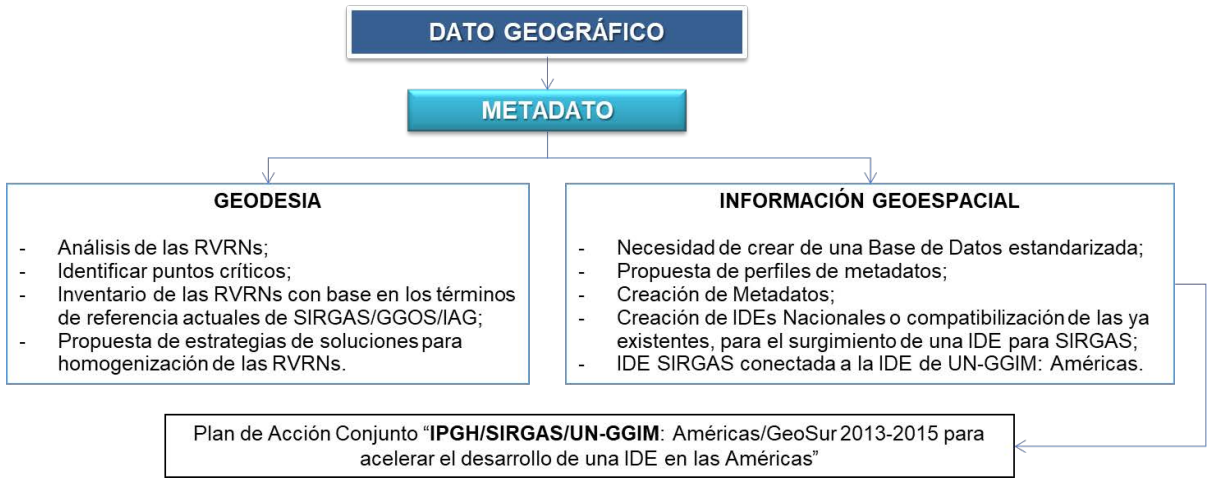

Figura 2. Uso de los metadatos.

Fuente: Santacruz, 2015. 


\section{Desarrollo metodológico y resultados}

En los países miembros de SIRGAS, se muestra la falta de creación de DataBase (DB) o las deficiencias existentes en estas, tales como: datos difusos entre diferentes organismos inclusive dentro de un mismo país; DB creadas parte con datos libres y parte con datos propietarios; datos con diferente calidad , confiabilidad o con calidad desconocida; entre otras. Como resultado, se evidencia la necesidad de crear un inventario de los datos geodésicos existentes, el cual será el primer paso para el desarrollo de una DB que contenga la información necesaria para contribuir en la toma de decisiones respecto a la definición del nuevo Sistema Vertical de Referencia SIRGAS.

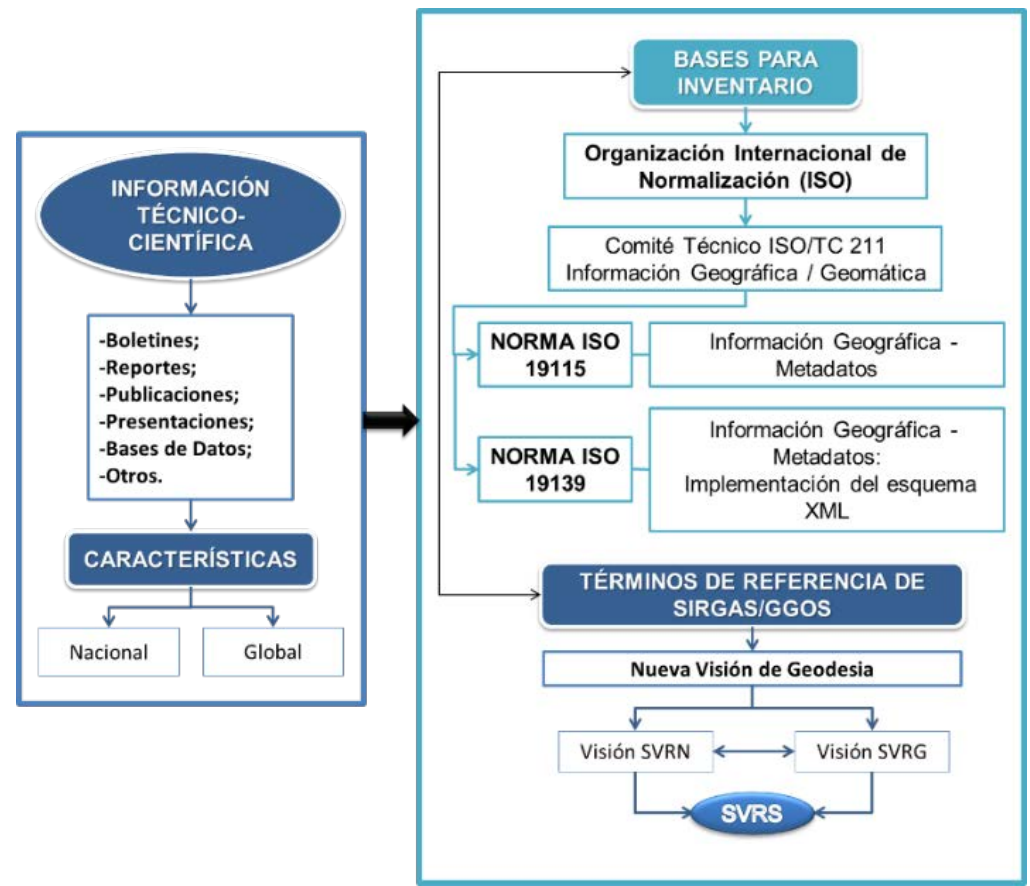

Figura 3. Esquema general de la metodología.

Fuente: Santacruz, 2015.

Para la realización del inventario, primeramente, se analizó el estado actual de los Sistemas Verticales existentes en la región SIRGAS, lo cual fue base para la recopilación de información técnica-científica de libre acceso que permita extraer características de los datos geodésicos nacionales y regionales. De esta forma se desarrolló una metodología basada en normas y estándares y que además puede ser aplicada para cualquier país (véase Figura 3). Como resultado, se generó Datos Geográficos clasificados según el análisis de las condiciones generales presentadas en los países pertenecientes a SIRGAS. Luego se los caracterizó como Tipo de Combinación de Datos con 
la información relevante de las redes, considerando los términos de referencia actuales de SIRGAS/GGOS/IAG (Figura 4). Además, se consideró las posibles combinaciones de estos datos en el terreno ya que se analizó a detalle la información que se extrajo. Este inventario contiene la información producida por el análisis general de las RVRNs de SIRGAS, el análisis de la información del caso de estudio realizado (RVR del Ecuador) y aspectos críticos respecto a la definición de redes nacionales, así como de las nuevas metodologías de establecimiento para ser discutidos. Con toda esta información recopilada en el inventario, se generó estrategias de solución para poder cumplir con el objetivo principal de esta investigación. Este estudio muestra algunas opciones de cómo se puede homogenizar los datos locales para una posible unificación, contribuyendo con estudios ya realizados. Además, puede ser adaptado en cada país, dependiendo de sus características locales.

\section{DATOS GEOGRÁFICOS}

\begin{tabular}{|c|c|c|c|}
\hline Datos Geográficos & \multirow{3}{*}{\multicolumn{2}{|c|}{ Descripción }} & $\Downarrow$ \\
\hline Estación Mareográfica & & & \multirow[b]{2}{*}{ Requisitos mínimos } \\
\hline Punto de Nivelación & & & \\
\hline Estación SIRGAS 1995 & \multirow{3}{*}{\multicolumn{3}{|c|}{ Conjunto de Datos Geográficos - CDG }} \\
\hline Estación SIRGAS 2000 & & & \\
\hline Estación SIRGAS-CON & & & \\
\hline Puntos GPS/GNSS & \multicolumn{3}{|c|}{$\downarrow$} \\
\hline Estaciones Gravimétricas & \multirow{4}{*}{\multicolumn{3}{|c|}{$\begin{array}{ll}\text { - } & \text { RGeRS: Red Geométrica de Referencia SIRGAS } \\
\text { - } & \text { DV: Datum Vertical; } \\
\text { - } & \text { RVR: Red Vertical de Referencia; } \\
\text { - } & \text { RS: Red Gravimétrica; } \\
\text { ASea sin información. }\end{array}$}} \\
\hline Puntos de Frontera & & & \\
\hline Área sin información & & & \\
\hline Otros & & & \\
\hline
\end{tabular}

Figura 4. Datos geográficos y sus conjuntos.

Fuente: Santacruz, 2015.

El dato geográfico, definido así en ISO (2014), por ejemplo "Estación Mareográfica" y "Punto de Nivelación" ayudaron a entender como fue establecida la red vertical y su materialización en el tiempo, permitiendo generar estrategias de solución para la actualización de los Conjuntos de Datos Geográficos (CDG) Datum Vertical del Ecuador (DVE) y Red Vertical de Referencia del Ecuador (RVRE). Los datos geográficos "Estación SIRGAS 1995”, "Estación SIRGAS 2000”, "Estación SIRGASCON" y "Punto GPS/GNSS" muestran el estado del Sistema Geométrico de Referencia SIRGAS-Ecuador (SGeRS-Ecuador), el cual sirve como componente geométrica para el SVRS y representa un CDG. El dato geográfico "Estación Gravimétrica” permitió conocer cómo se podría establecer una Red Gravimétrica del Ecuador (RGE), generando estrategias para su creación y/o actualización para este CDG. El dato “Área sin Información” fue caracterizado con la finalidad de localizar las áreas con poca densificación de datos o áreas que no tengan datos terrestres, para generar estrategias 
basadas en el PVCG (véase ecuación 1). para la obtención de datos a través de modernas herramientas como plataformas orbitales. Este es un punto relevante ya que se podría obtener en áreas de difícil acceso, soluciones basadas en MGGs y GNSS/gravedad sin depender de nivelación (véase ecuación 6). Por último, el dato "Punto de Frontera" sirve en el análisis de conexión entre países, para esto es indispensable la homogenización de datos y el trabajo conjunto basado en la misma metodología para enlazar un país con otro, mediante un sistema basado en números geopotenciales.

Para llegar a un mayor entendimiento respecto a la estructura de esta investigación, se lo considera como un Sistema en el cual se trabajó con datos geodésicos. Para esto se generó paralelamente Diagramas de Caso de Uso general (Véase Figura 5). Diagramas de Caso de Uso junto con Diagrama de Clases para Ecuador (Santacruz, 2015). Estos dan a conocer cómo se está observando el Sistema, tanto dentro como fuera de este.

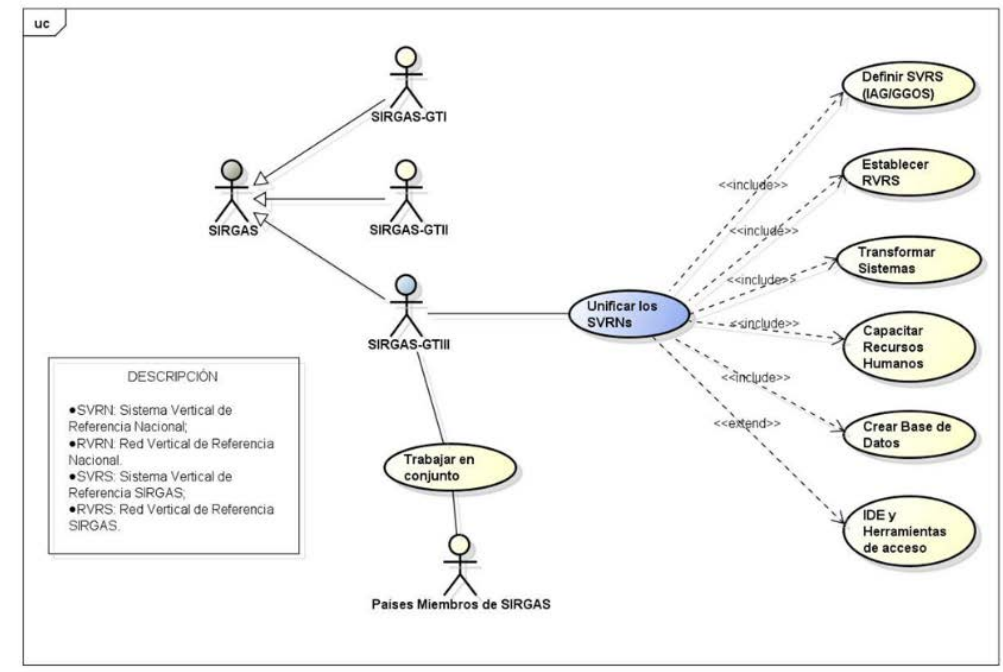

Figura 5. Diagramas de Caso de Uso.

Fuente: Santacruz, 2015.

Para el manejo de estos datos fue necesario el uso de la Norma ISO 19115 de la ISO/TC 211 Información Geográfica/Geomática (IPGH, 2010), permitiendo así, implantar el uso de metadatos organizados, estandarizados con una visión de creación de planillas de metadatos que contemplen las características importantes de las RVRNs. Fueron propuestos cuatro modelos de planillas de metadatos que corresponden al CDG levantados (Santacruz, 2015). 
En la Tabla 1 se muestra el resultado del CDG Red Vertical de Referencia del Ecuador, el cual concentra las características, avances, estrategias y posibles soluciones que se podrían aplicar para actualizar esta red, los demás CDGs pueden ser visualizados en Santacruz (2015).

\section{Tabla 1}

Análisis y estrategias de solución de la RVRE

\begin{tabular}{|c|c|}
\hline \multicolumn{2}{|r|}{ RED VERTICAL DE REFERENCIA DEL ECUADOR (RVRE) } \\
\hline DATO & Estación Mareográfica, Punto de Nivelación \\
\hline UBICACIÓN & Ecuador Continental \\
\hline \multirow{5}{*}{ 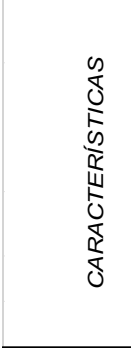 } & $\begin{array}{l}\text { - El origen de la Red de Control Básico Vertical del Ecuador se origina en la cota de marca de nivel "BM3". } \\
\text { Este se encuentra cerca de la estación mareográfica "La Libertad", la cual representa el DV de país; }\end{array}$ \\
\hline & $\begin{array}{l}\text { - La Red de Control Básico Vertical de primer orden cuenta con } 3.732 \text { puntos de nivelación a lo largo de los } \\
\text { principales ejes viales del país, los mismos inicialmente están distribuidos en } 62 \text { líneas, } 20 \text { anillos o circuitos } \\
\text { y } 38 \text { nodos. Cada línea cuenta con placa inicial, placa final, desnivel, distancia, cota y años (de 1977-2009) } \\
\text { en los que se realizó la nivelación. }\end{array}$ \\
\hline & $\begin{array}{l}\text { - No existe densificación de la Red en el Oriente Ecuatoriano debido al acceso restringido a esta zona (falta } \\
\text { de vías, zonas sin exploración, otros); }\end{array}$ \\
\hline & - Esta Red no se encuentra ajustada; \\
\hline & - Existen puntos nivelados que no se encuentran en los anillos de nivelación. \\
\hline \multirow{6}{*}{ 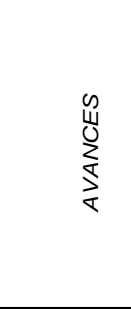 } & - Digitación de los datos en formato físico de la Red de Control Básico Vertical; \\
\hline & $\begin{array}{l}\text { - Estudio de un Ajuste de la Red de Control Básico Vertical en función de números geopotenciales, } \\
\text { considerando nuevos anillos de nivelación; }\end{array}$ \\
\hline & - Avances en el proyecto del IGM de remedición de los anillos fundamentales de la red vertical nacional; \\
\hline & - Continuidad de la nivelación y renivelación; \\
\hline & $\begin{array}{l}\text { - Identificación y digitación de todas las líneas de nivelación que conectan las estaciones SIRGAS2000: } \\
\text { (SIRGAS95 - Latacunga y Zamora -, mareógrafos -Libertad-y estaciones fronterizas -Tulcán y Zamora); }\end{array}$ \\
\hline & - Se realizó el cálculo de los números geopotenciales de los nodos de nivelación \\
\hline \multirow{8}{*}{ 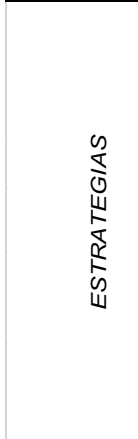 } & $\begin{array}{l}\text { - Antes del ajuste de la Red, analizar los errores de las alturas en las líneas de nivelación, y datos de } \\
\text { gravedad originales, considerando la distancia máxima entre puntos de nivelación y gravimetría, a más de las } \\
\text { condiciones topográficas; }\end{array}$ \\
\hline & - Ajuste de la red vertical en función de números geopotenciales; \\
\hline & - Revisión del Ajuste de la Red de nivelación, considerando los errores máximos de cierre; \\
\hline & $\begin{array}{l}\text { - Reajuste de los circuitos de nivelación que generen gran distorsión en la red durante su ajuste (ver los } \\
\text { errores de cierre de los circuitos); }\end{array}$ \\
\hline & - Densificación de líneas de nivelación en fronteras y en regiones amazónicas; \\
\hline & - Determinación de las diferencias de geopotencial; \\
\hline & - Determinación de diferencias en conexiones de frontera. \\
\hline & - Determinación de Números Geopotenciales para determinación de parámetros de conversión. \\
\hline $\begin{array}{l}\text { POSIBLE } \\
\text { SOLUCIÓN }\end{array}$ & $\begin{array}{l}\text { Obtener datos TIPO 6: (Nivelación + GPS (GNSS) geodésico L1/L2) + Gravimetría ya que con las redes de } \\
\text { nivelación y gravimétrica debidamente ajustadas, se podrá desarrollar la solución con base en Ihde (2007- } \\
\text { 2011) para la conexión regional y para la unificación de un SVRG. en las áreas sin información usar MGGs. }\end{array}$ \\
\hline
\end{tabular}

Fuente: Santacruz, 2015. 


\section{Conclusiones y recomendaciones}

Se creó una propuesta de inventario de metadatos considerando normas y convenciones con el objetivo de generar posibles estrategias de solución basados en los términos de referencia de SIRGAS/GGOS/IAG. Estas estrategias podrían considerar los problemas que se presentan en la homogenización de datos nacionales, conexiones entre países e integración de las redes verticales Sudamericanas. Por otra parte, gracias a la propuesta de trabajar con metadatos, se afianzó la necesidad de crear una IDE para SIRGAS, anticipando la homogenización de los datos y su almacenamiento, considerando así los objetivos de UN-GGIM: Américas. Por tanto, se puede concluir que este estudio tiene una contribución en el campo de la Geodesia y del manejo de Datos Geoespaciales, la cual se torna relevante y podría ser considerada por los países miembros de SIRGAS. Además, la metodología puede ser aplicada a cualquier país.

Con este estudio también se detectó y confirmó aspectos críticos respecto a las definiciones de las RVRNs, generando estrategias nacionales y continentales. Se concluye la importancia en el cálculo de números geopotenciales en cada país, considerando ajuste de las redes verticales y gravimétricas (forma convencional) y aplicando el PVCG en su forma fija para la determinación del potencial perturbador a través del disturbio de la gravedad (forma moderna) en áreas donde no hay datos de nivelación. Se considera también la determinación de las diferencias del geopotencial para el enlace entre países, independientemente del origen de las redes.

Un estudio semejante puede ser realizado por los demás países de SIRGAS, el cual aportará con mayor comprensión referente a las heterogeneidades de sus datos, directamente relacionadas con sus RVRNs. De esta forma se considera la nueva visión de SIRGAS-GTIII para conseguir la integración de las RVRNs y el establecimiento de una única Red Vertical de Referencia SIRGAS (RVRS).

\section{Agradecimientos}

Al Gobierno de la República del Ecuador, por darme la oportunidad de obtener la beca de estudios mediante la Secretaría de Educación Superior, Ciencia, Tecnología e Innovación (SENESCYT).

Al Curso de Pós-Graduação em Ciências Geodésicas - CPGCG de la Universidade Federal do Paraná (UFPR) - Brasil, especialmente a mi orientador Profesor Doctor Sílvio de Freitas e a mi co-orientadora la Profesora Doctora Silvana Camboim.

Al Sistema de Referencia Geocéntrico para las Américas (SIRGAS) y a su Grupo de Trabajo III: Datum Vertical. 


\section{Bibliografía}

Angermann, D.; Gruber, T.; Gerstl, M.; Heinkelmann, R.; Hugentobler, U.; Sánchez, L., and Steigenberger, P., (2016). "GGOS Bureau of Products and Standards Inventory of Standards and Conventions used for the Generation of IAG Products”. Journal of Geodesy: The Geodesist's Handbook 2016, 90 (10), pp. 1095-1156.

Brovar, V., (1972). "A possible improvement in the accuracy of gravimetric results in Geodesy”, Sov. Astron, 15, pp. 1055-1058.

De Freitas, S.R.C.; Medina, A.S.; Lima, S.R.S., (2002). "Associated problems to link South American Vertical Networks and possible approaches to face them”, in: Drewes, H.; Dodson, A.H.; Fortes, L.P.S.; Sánchez, L.; Sandoval, P. (ed.). Vertical Reference Systems. International Association of Geodesy Symposium (IAGS), vol. 124, Springer, Verlag, Berlim, pp. 318323.

De Freitas, S.R.C., (2015). "SIRGAS-WGIII activities for unifying height systems in Latin America”, Revista Cartográfica, no. 91, p. 75.

Drewes, H.; Sánchez, L.; Blitzkow, D.; Freitas, S., (2002). "Scientific foundations of the SIRGAS vertical reference system”, in: Drewes et al. Eds. IAG Symposia 124, Springer, pp. 297-301.

Drewes, H.; Kuglitsch, F.; Ádám, J.; Rózsa, S., (2016). “The geodesist’s handbook 2016”, Journal of Geodesy 90.10, pp. 907-1205. DOI: 10.1007/s00190016-0948-z.

Gemael, C., (2002). "Introdução à Geodésia Física”, Curso de Pós-graduação em Ciências Geodésicas, Universidade Federal do Paraná, Curitiba, 1999, reimpressão em 2002.

Heck, B., (2011). "A Brovar-type solution of the fixed geodetic boundary - value problem”, Studia Geophysica et Geodaetica, vol. 55, n. 3, pp. 441-454.

Hofmann-Wellenhof, B.; Moritz, H., (2005). "Physical Geodesy”, Springer Wien New York.

IAG (International Association of Geodesy). Acceso en: 2014. "The Structure of IAG (2011-2015)”. Disponible en: http://www.iag-aig.org/ index.php?tpl=text\&id_c=12\&id_t=516.

Ihde, J., (2007). "IAG-ICP 1.2. Inter-Commission Project 1.2: Vertical Reference Frames", Report of the International Association of Geodesy (20072011).

Ihde, J.; Barzaghi, R.; Marti, U.; Sánchez, L.; Sideris, M.; Drewes, H.; Foerste, C.; Gruber, T.; Liebsch, G.; Pail, R., (2015). "Report of the ad hoc group on an International Height Reference System (IHRS)", in: Drewes H, Hornik H. (eds.), Travaux de l'AIG 39, IAG Reports, pp. 2011-2015.

IPGH (Instituto Panamericano de Geografía e Historia), (2010) "Guía De Normas, Edición en español, Comité ISO/TC211 Información Geográfica/Geomática”. Grupo Consultivo de Desarrollo, Ex Arzobispado 29, Colonia Observatorio, 11860 México, D.F. 
ISO/TC 211 (International Organization for Standardization. Geographic information/Geomatics). (2003). “About ISO/TC 211”. Disponible en: <http:// www.isotc211.org>, acceso en: 2014.

Kutterer H.; Neilan R.; Bianco G., (2012). "Global Geodetic Observing System (GGOS)”, in: Drewes H., H. Hornik, J. Ádám, S. Rózsa (Eds. 2012). The geodesist's handbook 2012. Journal of Geodesy, 86 (10), pp. 915-926. DOI: $10.1007 / \mathrm{s} 00190-012-0584-1$.

Plag, H.-P.; Pearlman, M., (2009). “Introduction of GGOS”. Global Geodetic Observing System - GGOS 2020 Book, Springer.

Sánchez, L. (2009). "Strategy to Establish a Global Vertical Reference System". Geodetic Reference Frames, Springer.

Sánchez L.; R. Luz, (2011). "Requerimientos para la unificación de los sistemas de alturas existentes en la Región SIRGAS”, presentado en la Reunión SIRGAS2011, Heredia, Costa Rica, agosto 8-10, 2011.

Sánchez, L.; Sideris, M.G., (2017). "Vertical datum unification for the International Height Reference System (IHRS)”. Geophysical Journal International, 209 (2), pp. 570-586.

Santacruz, A., (2015). "Integración de las Redes Verticales Sudamericanas: Inventario en vista de los términos de referencia de SIRGAS/GGOS”. Disertación de Maestría en Ciencias Geodésicas. Universidad Federal de Paraná.

Santacruz Jaramillo, A.; Freitas, S.R.C.; Camboim, S. "Integração das Redes Verticais Sul-Americanas: Inventário em vista dos termos de Referência do SIRGAS/IAG/GGOS”, Revista Brasileira de Cartografia (Online), 67, pp. 1279-1295.

SIRGAS (Sistema de Referencia Geocéntrico para las Américas). Acceso en 2014. "Unificación de los sistemas de alturas existentes". Disponible en: $<$ http://www.sirgas.org/index.php?id=75>.

UN-GGIM: Américas, “Acerca de UN-GGIM: Américas”. Disponible en <http://www.cp-idea.org/index.php/un-ggim-americas/acerca-de-unggim-americas>, acceso en: 2014.

UN-GGIM, (2013). "Statutes of UN-GGIM: Americas”. Regional Committee of United Nations on Global Geospatial Information Management for the Americas. 